\title{
Training Need Assessment of Tasar Silkworm Rearing Farmers in Chhattisgarh
}

\author{
Kedar Nath Yadaw ${ }^{1 *}$ and M. L. Sharma ${ }^{2}$ \\ ${ }^{1}$ Krishi Vigyan Kendra, Balod, India \\ ${ }^{2}$ Department of Agricultural Extension, IGKV Raipur (C.G.), India \\ *Corresponding author
}

\section{A B S T R A C T}

\section{Keywords}

Need assessment, Training needs, Silkworm rearing farmers, Tasar silkworm rearing, Chhattisgarh

\section{Article Info}

Accepted: 20 December 2020 Available Online: 10 January 2021
The present study was conducted in 18 selected villages from the Raigarh and Korba districts of Chhattisgarh with 270 randomly selected sericulturists to assess the training need towards improved tasar silkworm rearing practices. The data were collected personally from sericulturists by using pre-tested and well structured interview schedule. The collected data were analyzed by using suitable statistical tools. The results of this study revealed that majority $(61.85 \%)$ of the respondents had not attended training programme whereas, 38.15 per cent of the respondents had attended training programme related to tasar silk cultivation. The training need of sericulturists was observed in to two specific areas which contribution of host plant cultivation and rearing practices of silkworm. According the findings shows that majority of the respondents perceived to get trained about plant protection with a mean score of 2.41 . Regarding rearing practices of silkworm the findings reveals that majority of the farmers were interested to attend the training programme related to processing of cocoons with a mean score of 2.60 followed by management of diseases and insect pest with mean score 2.55 . The extent of training need also computed which indicates that 38.89 per cent respondents extremely needed the training programme of improved tasar silk cultivation practices followed by 30.74 per cent respondents most need the training. While 15.92 per cent sericulturists having little need of training about improved practices of silkworm rearing. Drastically about 14 per cent of the respondents were not shown any interest for attending the training programme on the tasar silk production technology. In this way it is required to organized strategic training programmes for aspirant's tasar silkworm rearing farmers for increasing it productivity and profitability.

\section{Introduction}

Sericulture, the production of silk, is an important social industry in the economy of our country. It provides employment to approximately 7.63 million persons
(Kushwaha and Singhavi, 2013). Sericulture plays a vital role in rural development, as it integrates with the farming systems and has the potential to generate attractive income throughout the year. It needs low capital and provides year round employment 
(Hanumappa and Erappa, 1985). India stands second in raw silk production after China in world scenario.

Sericulture is an eco-friendly, agro-based labour intensive rural cottage industry providing subsidiary employment and supplementing the income of rural families especially the economically weaker section of the society.

The industrial production of raw silk and fabric employs a large number of semi literate and semi skilled poor workers throughout the year. Thus, a large portion of the cost incurred in the conversion of soil to silk reaches poor workers involved in the value addition at each stage like cocoon production, silk reeling, fabric production, dying and printing.

Sericulture industry is therefore, discretely helping in building an egalitarian society in highly populous countries like India and China. Sericulture plays an important role in transformation of rural economy as it assures regular employment and periodic returns round the year (Lakshmannan et al., (1998)).

There are more than 58 countries practicing sericulture in the world. India is the only Country in the world to produce all the four known varieties of silk including Mulberry, Eri, Tasar and Muga. In India cultivation is spread over 22 states, covering 172000 hectares across 54000 villages operating 258000 handlooms and 29340 powers looms (Dewangan, 2010). In India, sericulture is not only a tradition but also a living culture. It particularly suits rural-based farmers, entrepreneurs and artisans, as it requires low investment but, with potential for relatively higher returns. It provides income and employment to the rural poor especially farmers with small land-holdings and the marginalized and weaker sections of the society. The need of the time is to enhance the production and productivity of this enterprise in the state.

Keeping in view of the above facts in to consideration, the present study was undertaken to assess the training need of silkworm rearing farmers in Chhattisgarh.

\section{Materials and Methods}

Two districts of Chhattisgarh state namely Raigarh and Korba were selected purposively for the study since they had the highest area and respondents under tasar silk cultivation. From the each selected district, 3 blocks (3X2 $=6$ ) were selected purposively for the study on the basis of highest area covered under sericulture. From each selected block, 3 villages $(3 \times 6=18)$ were selected on the basis of maximum availability of sericulturist in the villages. From each selected village, 15 sericulturists were selected randomly by using simple random sample method.

In this way a total of 270 sericulturists were considered as respondent for the present study. Primary data from sericulturists were collected though personal interview with the help of pre-tested structured interview schedule. Collected data were tabulated and processed by using appropriated statistical tools i.e frequency, percentage etc.

The training need of sericulturists in these two main areas namely; host plant cultivation and silkworm rearing of improved tasar silk cultivation were obtained, in order to ascertain the extent of training need of sericulturists in tasar silk cultivation.

The training need of sericulturists were recorded in to four point continuum scale, namely "most needed", "needed", "somewhat needed" and "not needed" by giving score ' 3 ', '2', ' 1 ' and ' 0 ', respectively. 
After calculating the total scores and mean scores of each item, the rank values were assigned and for preference of training in a particular area the below mentioned scale was adopted:

\begin{tabular}{|l|c|c|c|}
\hline Most needed & $(\mathbf{M N})$ & $\mathbf{0 2 . 2 6}-\mathbf{0 3 . 0 0}$ & Mean score \\
\hline Needed & $(\mathrm{N})$ & $01.51-02.25$ & Mean score \\
\hline Somewhat needed & $(\mathrm{SN})$ & $00.76-01.50$ & Mean score \\
\hline Not needed & $(\mathrm{NN})$ & $00.00-00.75$ & Mean score \\
\hline
\end{tabular}

\section{Results and Discussion}

Training participation the sericulturist about various aspects of sericultural technology

The data regarding training received by sericulturist are presented in Table 1. The data shows that majority $(61.85 \%)$ of the respondents had not attended training programme whereas, 38.15 per cent of the respondents had attended training programme in tasar silk cultivation. Manju (1997), Reddy (2006), Geetha and Devi (2008) and Vindhya et al., (2012) found almost similar findings in their study.

The data regarding frequency of participation in training programme are evident in Table 1. The results reveal that out of total trained sericulturist, 52.53 per cent of them participated in training programme only once, followed by 38.83 per cent of the sericulturists participated thrice or above and only 08.74 per cent of the sericulturists participated training programme twice.

The data regarding duration of training programme attained are evident in Table 1. The results reveal that out of total trained sericulturist, 69.90 per cent of the sericulturists were involved in training for more than 1 week, followed by 11.65 per cent of sericulturists were attended training of one week, 10.68 per cent of sericulturists had taken training of 2 to 4 days, while only 7.77 per cent of sericulturists were involved in one day training programme. Reddy (2006), Geetha and Devi (2008) found almost similar findings in their studies.

The data regarding utility of training programme indicated that, 50.49 per cent of the respondents expressed training are moderately utilized, followed by 43.69 per cent of the respondents reported that training programme is fully utilized, while only 5.82 per cent of the respondents reported that training programme were not utilized.

Training need in sub-area of land preparation

Table 2 indicated the training needs about treatment of pit for termite control (m.s. 1.47) was reported by sericulturist in their highest priority. Whereas, preparation of pit for host plants was given second rank. Similarly, selection of land/ soil and field sanitation and labelling were ranked III and IV with m.s. 0.58 and 0.44 , respectively.

Training need in sub-area of plat propagation

Further, Table 2 shows the training need of the respondents in sub-area of plant propagation. It reveals the priority wise need of training of respondents i.e., vegetative propagation of host plants (I rank), ratio of soil, sand and manures in polythene bags (II rank), chawki and main field plantation (III rank), sowing of seeds in polythene bags (IV rank), preparation of bund of water treated 
seeds (V rank), water treatment of seeds (VI rank), selection of healthy seeds (VII rank), and collection of seeds and management of nursery (VIII rank), respectively.

\section{Training need in sub-area of handling of host plants}

Training needs related to with sub-areas of handling of host plants is presented in Table 3. As per the perception of respondents, use of manures and fertilizers got a maximum 1.92 mean score, hence ranked first. Foliar spray of urea (m.s. 1.88), quantity of manures and fertilizers (m.s. 1.83), training and pruning of host plants (m.s. 1.52), preparation of rings for irrigations (m.s. 0.76), irrigation (m.s. 0.29) and intercultural operations (m.s. 0.28) were ranked II, III, IV, V, VI, and VII, respectively.

\section{Training need in sub-area of application FYM and fertilizers}

The data compiled in Table 3 further shows the training need of respondents in the field of sub-area of application of FYM and fertilizers. It reveals that calculation of price of per units of fertilizers got maximum mean score 2.38, hence perceived first by the respondents. Collection of soil samples (m.s. 2.36), related to required quantity of fertilizers (m.s. 2.26), methods of application of fertilizers (m.s. 2.21), foliar spray of urea (m.s. 2.13) and identification of fertilizers (m.s. 2.02) were ranked II, III, IV, V and VI, respectively.

\section{Training need in sub-area of plant protection}

With regards to training need on sub-area of plant protection, table 3 reveals, that the important areas like identification of insect pests and diseases on host plant were ranked first, having 2.49 mean score. Whereas, the respondents were given second priority of training need towards the use of insecticide and fungicide. Insecticide and fungicide to control insect pest and diseases and use of sprayers and dusters, were found as the III and IV ranking training needs of the respondents.

Training need in sub-area of basic practices and handling of eggs

With regards to training need on sub-areas of basic practices and handling of eggs, table 4 reveals, that the important areas like ratio of formalin or H.C.L. solution for disinfection of tasar silkworm eggs were ranked first, having 2.72 mean score. Whereas, the respondents were given second priority of training need about the temperature and humidity for hatching of tasar silkworm eggs. Selection of improved species of silk worm, use of equipments and material required in silk worm rearing, and Eggs for tasar silk worm rearing were found as the III, IV and V ranking training needs of the respondents.

Training need in sub-area of chawki silkworm rearing for tasar silkworm rearing

Training needs of the respondents with subarea of chawki silkworm rearing is also shown in table 4. It reveals that respondents ranked first need of training in the field of size of nylon net and methods covering host plants in the chawki garden with mean score 2.17. Size of brushing bag were ranked second with 1.97 mean score, whereas, use of brushing bags in the plants, brushing of larvae in the chawki garden, methods of brushing larvae in chawki garden, stages of silkworms in chawki garden and brushing of larvae in the host plants, respectively were ranked III, IV, V, VI and VII with 1.93, 1.88, 1.84, and 1.81 mean scores, respectively. 
Training need in sub-area of adult stage silkworm rearing

The data compiled in table 5 reveals the training needs of the respondents in sub-areas of adult stage silkworm rearing. It was found that the respondents needed training on disinfections of field by spreading of lime and bleaching powder before transfer of silkworms (m.s. 2.51) followed by foliar spray of urea solution before transfer of silkworms on the host plants. Transfer of silkworms in economic plants from chawki garden and brushing of silkworms in the host plant, respectively were ranked II, III and IV, respectively for attaining training.

Training need in sub-area of disease and insect control

Training needs of respondents in sub-areas of disease and insect control are shown in Table 5. It reveals the priority wise training need of respondents in the field of use of bio-pesticide and insecticide (m.s. 2.81), name, quantity and methods of using insecticide and fungicide (m.s. 2.75), time of using insecticide and fungicide (m.s. 2.73), about attack of insect pest and diseases at larval and adult stage of silkworm rearing (m.s. 2.68), ways to check pests (e.i. ants, lezzard, rates, etc.) to attack in the host plants (m.s. 2.59), about attack of parasite and predators at larval and adult stage of silkworm rearing (m.s. $2.41)$, control attack of insects in the storage house (m.s. 2.21) and use of sprayers and dusters (m.s. 2.18).

\section{Training need in sub-area of harvesting and storage of cocoons}

The training need in sub-area of harvesting and storage of cocoons is shown in Table 6. It reveals that the first ranked training need of respondents were commercial storage cocoons. Whereas, the II, III, IV, V and VI ranks were given to preparation of chain of cocoons, methods of storage of cocoons after harvesting, selection of healthy cocoons, methods of cutting cocoons and best rime for harvesting of cocoons, respectively.

\section{Training need in sub-area of processing of cocoons}

Training needs in sub-areas of processing of cocoons is presented in the Table 6. It reveals that methods of reeling got maximum 2.72 mean score, hence ranked first by the respondents. Reeling of cocoons and preparation of products (m.s. 2.71), grading of cocoons (m.s. 2.63) and boiling of cocoons (m.s. 2.22) were ranked II, III and IV, respectively.

\section{Training need of sericulturist in the main area of tasar silk cultivation}

Training need of sericulturist in the tasar silk cultivation is divided in two main areas i.e. host plant cultivation and silkworm rearing. The data regarding both main areas are compiled in Table 7. As regards to training needs in the area of host plant cultivation, the data reveals that the training needs of respondents in the field of plant protection is got maximum 2.41 mean score, hence first ranked by the respondents. Application of FYM and fertilizers (m.s. 2.22), handling of host plants (m.s. 1.21), plant propagation (m.s. 1.09) and land preparation (m.s. 0.83) were ranked II, III, IV and V ranked, respectively. Prasad et al., (2010) and Vindhya et al., (2012) expressed almost similar findings in their study.

Regarding silkworm rearing, the data reveals that processing of cocoons got maximum 2.60 mean score, hence ranked first by the respondents. Disease and insect control (m.s. 2.55), basic practices and handling of eggs (m.s. 2.50), chawki silkworm rearing (m.s. 
1.93) and harvesting and storage of cocoons (m.s. 1.11) were ranked II, III, IV, V and VI, respectively. Patel and Veerbhadraiah (1987), Ranganatha et al., (2003) and Shivamurthy et al., (2003), Geetha and Devi (2008), Mech et al., (2010) and Vindhya et al., (2012) found almost similar findings in their studies.

The data regarding overall training needs about of tasar silk cultivation are evident in Table 8. This table indicated that the most of the sericulturist $(38.89 \%)$ were needed training programme, followed by most needed $(30.74 \%)$ and somewhat needed (15.92\%). While 14.45 per cent of sericulturists were not needed training on tasar silkworm rearing. Gurav et al., (2010) observed almost similar findings in their study on training needs of Agriculture Assistants from Single Window System in Maharashtra. Verma et al., (2013) also found almost similar findings in their study.
Perceived preferences of farmers regarding organization of training programme

The data regarding various aspects of training preference is evident from Table 9. Data in respect of preference of place of training indicated that majority of the respondents $(58.15 \%)$ preferred to conduct training programmes at village level. On the other hand, 26.30 per cent of the respondents desired to have training at exhibition centre and 8.51 per cent of the respondents desired to have training at research station, while 7.04 per cent respondents wanted to attain training at the nearest research extension centre/ departmental farm. Mech et al., (2013) had doing almost similar study on training need of rural women in Eri culture in Assam Province. Geetha and Devi (2008) and Paswan et al., (2013) give almost similar findings in their studies.

Table.1 Distribution of respondents according to their participating in training programme $(\mathrm{n}=270)$

\begin{tabular}{|c|c|c|}
\hline Particulars & Frequency & Percentage \\
\hline \multicolumn{3}{|l|}{ Participation } \\
\hline$>$ Attended & 103 & 38.15 \\
\hline$>$ Not attended & 167 & 61.85 \\
\hline \multicolumn{3}{|l|}{ Frequency of participation } \\
\hline$>$ Participated once & 54 & 52.43 \\
\hline$>$ Participated twice & 09 & 08.74 \\
\hline$>$ Participated thrice and above & 40 & 38.83 \\
\hline \multicolumn{3}{|l|}{ Duration of training } \\
\hline$>$ One day & 08 & 07.77 \\
\hline$>2$ to 4 days & 11 & 10.68 \\
\hline$>1$ week & 12 & 11.65 \\
\hline$>$ More than 1 week & 72 & 69.90 \\
\hline \multicolumn{3}{|l|}{ Adoptability of training programme } \\
\hline$>$ Fully utilized & 45 & 43.69 \\
\hline$>$ Moderately utilized & 52 & 50.49 \\
\hline$>$ Not utilized & 06 & 05.82 \\
\hline
\end{tabular}


Table.2 Training needs in sub-areas of land preparation and plant propagation $(\mathrm{n}=270)$

\begin{tabular}{|c|c|c|c|c|c|c|c|c|}
\hline \multirow[t]{2}{*}{ Sub-areas } & \multicolumn{4}{|c|}{ Training need } & \multirow[t]{2}{*}{ TS } & \multirow[t]{2}{*}{ MS } & \multirow[t]{2}{*}{ Rank } & \multirow[t]{2}{*}{$\mathbf{C P}$} \\
\hline & $\begin{array}{c}\text { MN } \\
(\mathrm{F} / \%)\end{array}$ & $\begin{array}{c}\mathrm{N} \\
(\mathbf{F} / \%)\end{array}$ & $\begin{array}{c}\text { SW } \\
(\mathbf{F} / \%)\end{array}$ & $\begin{array}{c}\text { NN } \\
(\mathbf{F} / \%)\end{array}$ & & & & \\
\hline \multicolumn{9}{|l|}{ Land preparation } \\
\hline 1. Selection of land/ soil & $\begin{array}{c}04 \\
(1.48)\end{array}$ & $\begin{array}{c}27 \\
(10.00)\end{array}$ & $\begin{array}{c}92 \\
(34.07)\end{array}$ & $\begin{array}{c}147 \\
(54.45)\end{array}$ & 158 & 00.58 & III & $\mathrm{NN}$ \\
\hline 2. Field sanitation and labelling & $\begin{array}{c}03 \\
(1.11)\end{array}$ & $\begin{array}{c}24 \\
(08.89)\end{array}$ & $\begin{array}{c}63 \\
(23.33)\end{array}$ & $\begin{array}{c}180 \\
(66.67)\end{array}$ & 120 & 00.44 & IV & $\mathrm{NN}$ \\
\hline $\begin{array}{l}\text { 3. Preparation of pit for host } \\
\text { plants }\end{array}$ & $\begin{array}{c}05 \\
(01.85)\end{array}$ & $\begin{array}{c}52 \\
(19.26)\end{array}$ & $\begin{array}{c}101 \\
(37.41)\end{array}$ & $\begin{array}{c}112 \\
(41.48)\end{array}$ & 220 & 00.81 & II & $\mathrm{SN}$ \\
\hline $\begin{array}{l}\text { 4. Treatment of pit for termite } \\
\text { control }\end{array}$ & $\begin{array}{c}39 \\
(14.44)\end{array}$ & $\begin{array}{c}97 \\
(35.93)\end{array}$ & $\begin{array}{c}87 \\
(32.22)\end{array}$ & $\begin{array}{c}47 \\
(17.41)\end{array}$ & 398 & 01.47 & I & $\mathrm{SN}$ \\
\hline \multicolumn{9}{|l|}{ Plant propagation } \\
\hline $\begin{array}{l}\text { 1. Vegetative (gutty and } \\
\text { grafting) propagation of host } \\
\text { plants }\end{array}$ & $\begin{array}{c}87 \\
(32.22)\end{array}$ & $\begin{array}{c}146 \\
(54.07)\end{array}$ & $\begin{array}{c}13 \\
(04.82)\end{array}$ & $\begin{array}{c}24 \\
(08.89)\end{array}$ & 566 & 02.09 & I & $\mathrm{N}$ \\
\hline 2. Collection of seeds & $\begin{array}{c}07 \\
(02.60)\end{array}$ & $\begin{array}{c}20 \\
(07.41)\end{array}$ & $\begin{array}{c}69 \\
(25.55)\end{array}$ & $\begin{array}{c}174 \\
(64.44)\end{array}$ & 130 & 00.48 & VIII & $\mathrm{NN}$ \\
\hline 3. Selections of healthy seeds & $\begin{array}{c}04 \\
(1.48)\end{array}$ & $\begin{array}{c}46 \\
(17.03)\end{array}$ & $\begin{array}{c}70 \\
(25.93)\end{array}$ & $\begin{array}{c}150 \\
(55.56)\end{array}$ & 174 & 00.64 & VII & $\mathrm{NN}$ \\
\hline 4. Water treatment of seeds & $\begin{array}{c}06 \\
(02.22)\end{array}$ & $\begin{array}{c}49 \\
(18.15)\end{array}$ & $\begin{array}{c}98 \\
(36.30)\end{array}$ & $\begin{array}{c}117 \\
(43.33)\end{array}$ & 214 & 00.79 & VI & $\mathrm{SN}$ \\
\hline $\begin{array}{l}\text { 5. Preparation of bund of water } \\
\text { treated seeds }\end{array}$ & $\begin{array}{c}03 \\
(01.11)\end{array}$ & $\begin{array}{c}62 \\
(22.96) \\
\end{array}$ & $\begin{array}{c}88 \\
(32.60) \\
\end{array}$ & $\begin{array}{c}117 \\
(43.33) \\
\end{array}$ & 221 & 00.82 & $\mathrm{~V}$ & SN \\
\hline $\begin{array}{l}\text { 6. Sowing of seeds in polythene } \\
\text { bags }\end{array}$ & $\begin{array}{c}07 \\
(02.59)\end{array}$ & $\begin{array}{c}101 \\
(37.41)\end{array}$ & $\begin{array}{c}84 \\
(31.11)\end{array}$ & $\begin{array}{c}78 \\
(28.89)\end{array}$ & 307 & 01.14 & IV & $\mathrm{SN}$ \\
\hline $\begin{array}{l}\text { 7. Ratio of soil, sand and } \\
\text { manures in polythene bags }\end{array}$ & $\begin{array}{c}45 \\
(16.67)\end{array}$ & $\begin{array}{c}159 \\
(58.89)\end{array}$ & $\begin{array}{c}58 \\
(21.48)\end{array}$ & $\begin{array}{c}08 \\
(02.96)\end{array}$ & 511 & 01.89 & II & $\mathrm{N}$ \\
\hline 8. Management of nursery & $\begin{array}{c}06 \\
(02.22)\end{array}$ & $\begin{array}{c}21 \\
(07.78)\end{array}$ & $\begin{array}{c}70 \\
(25.93)\end{array}$ & $\begin{array}{c}173 \\
(64.07)\end{array}$ & 130 & 00.48 & VIII & $\mathrm{NN}$ \\
\hline $\begin{array}{l}\text { 9. Chawki and main field } \\
\text { plantation }\end{array}$ & $\begin{array}{c}31 \\
(11.48)\end{array}$ & $\begin{array}{c}110 \\
(40.74)\end{array}$ & $\begin{array}{c}84 \\
(31.11)\end{array}$ & $\begin{array}{c}45 \\
(16.67)\end{array}$ & 397 & 01.47 & III & SN \\
\hline
\end{tabular}


Table.3 Training needs in sub-areas of handling of host plants, application of FYM and fertilizers and plant protection measure $(n=270)$

\begin{tabular}{|c|c|c|c|c|c|c|c|c|}
\hline \multirow{2}{*}{ Sub-areas } & \multicolumn{4}{|c|}{ Training need } & \multirow[t]{2}{*}{ TS } & \multirow[t]{2}{*}{ MS } & \multirow{2}{*}{ Rank } & \multirow[t]{2}{*}{$\mathbf{C P}$} \\
\hline & $\begin{array}{c}\text { MN } \\
(\mathrm{F} / \%)\end{array}$ & $\begin{array}{c}\mathbf{N} \\
(\mathbf{F} / \%)\end{array}$ & $\begin{array}{c}\text { SN } \\
(\mathbf{F} / \%)\end{array}$ & $\begin{array}{c}\text { NN } \\
(\mathbf{F} / \%)\end{array}$ & & & & \\
\hline \multicolumn{9}{|l|}{ Handling of host plants } \\
\hline 1. Irrigation & $\begin{array}{c}03 \\
(01.11)\end{array}$ & $\begin{array}{c}12 \\
(04.44)\end{array}$ & $\begin{array}{c}46 \\
(17.04)\end{array}$ & $\begin{array}{c}209 \\
(77.41)\end{array}$ & 79 & 00.29 & VI & NN \\
\hline 2. Intercultural operations & $\begin{array}{c}02 \\
(00.74)\end{array}$ & $\begin{array}{c}18 \\
(06.67)\end{array}$ & $\begin{array}{c}35 \\
(12.96)\end{array}$ & $\begin{array}{c}215 \\
(79.63)\end{array}$ & 77 & 00.28 & VII & NN \\
\hline $\begin{array}{l}\text { 3. Preparation of ring for } \\
\text { irrigations }\end{array}$ & $\begin{array}{c}07 \\
(02.59)\end{array}$ & $\begin{array}{c}50 \\
(18.52)\end{array}$ & $\begin{array}{c}86 \\
(31.85)\end{array}$ & $\begin{array}{c}127 \\
(47.04)\end{array}$ & 207 & 00.76 & V & $\mathrm{SN}$ \\
\hline $\begin{array}{l}\text { 4. Quantity of manures and } \\
\text { fertilisers }\end{array}$ & $\begin{array}{c}28 \\
(10.37)\end{array}$ & $\begin{array}{c}185 \\
(68.52)\end{array}$ & $\begin{array}{c}41 \\
(15.18)\end{array}$ & $\begin{array}{c}16 \\
(05.93)\end{array}$ & 495 & 01.83 & III & $\mathrm{N}$ \\
\hline $\begin{array}{l}\text { 5. Use of manures and } \\
\text { fertilisers }\end{array}$ & $\begin{array}{c}30 \\
(11.11)\end{array}$ & $\begin{array}{c}193 \\
(71.48)\end{array}$ & $\begin{array}{c}42 \\
(15.56)\end{array}$ & $\begin{array}{c}05 \\
(01.85)\end{array}$ & 518 & 01.92 & I & $\mathrm{N}$ \\
\hline 6. Foliar spray of urea & $\begin{array}{c}35 \\
(12.96)\end{array}$ & $\begin{array}{c}177 \\
(65.56)\end{array}$ & $\begin{array}{c}49 \\
(18.15)\end{array}$ & $\begin{array}{c}09 \\
(03.33)\end{array}$ & 508 & 01.88 & II & $\mathrm{N}$ \\
\hline $\begin{array}{l}\text { 7. Training and pruning of } \\
\text { host plants }\end{array}$ & $\begin{array}{c}17 \\
(06.30)\end{array}$ & $\begin{array}{c}141 \\
(52.22)\end{array}$ & $\begin{array}{c}77 \\
(28.52)\end{array}$ & $\begin{array}{c}35 \\
(12.96)\end{array}$ & 410 & 01.52 & IV & $\mathrm{N}$ \\
\hline \multicolumn{9}{|c|}{ Application of FYM and fertilizers } \\
\hline $\begin{array}{l}\text { 1. Identification } \\
\text { fertilizers }\end{array}$ & $\begin{array}{c}60 \\
(22.22)\end{array}$ & $\begin{array}{c}164 \\
(60.74)\end{array}$ & $\begin{array}{c}38 \\
(14.08)\end{array}$ & $\begin{array}{c}08 \\
(2.96)\end{array}$ & 546 & 2.02 & VI & $\mathrm{N}$ \\
\hline $\begin{array}{l}\text { 2. Calculation of price of } \\
\text { per units of fertilizers }\end{array}$ & $\begin{array}{c}112 \\
(41.48)\end{array}$ & $\begin{array}{c}151 \\
(55.93)\end{array}$ & $\begin{array}{c}06 \\
(02.22)\end{array}$ & $\begin{array}{c}01 \\
(00.37)\end{array}$ & 644 & 02.38 & I & MN \\
\hline $\begin{array}{l}\text { 3. Related to required } \\
\text { quantity of fertilizers }\end{array}$ & $\begin{array}{c}83 \\
(30.74)\end{array}$ & $\begin{array}{c}176 \\
(65.19)\end{array}$ & $\begin{array}{c}10 \\
(03.70)\end{array}$ & $\begin{array}{c}01 \\
(00.37)\end{array}$ & 611 & 02.26 & III & MN \\
\hline 4. Collection of soil samples & $\begin{array}{c}102 \\
(37.78)\end{array}$ & $\begin{array}{c}164 \\
(60.74)\end{array}$ & $\begin{array}{c}04 \\
(01.48)\end{array}$ & $\begin{array}{c}00 \\
(00.00)\end{array}$ & 638 & 02.36 & II & MN \\
\hline $\begin{array}{l}\text { 5. Method of application of } \\
\text { fertilizers }\end{array}$ & $\begin{array}{c}81 \\
(30.00)\end{array}$ & $\begin{array}{c}167 \\
(61.85)\end{array}$ & $\begin{array}{c}22 \\
(08.15)\end{array}$ & $\begin{array}{c}00 \\
(00.00)\end{array}$ & 599 & 02.21 & IV & $\mathrm{N}$ \\
\hline 6. Foliar spray of urea & $\begin{array}{c}75 \\
(27.78)\end{array}$ & $\begin{array}{c}159 \\
(58.89)\end{array}$ & $\begin{array}{c}34 \\
(12.59)\end{array}$ & $\begin{array}{c}02 \\
(00.74)\end{array}$ & 577 & 02.13 & $\mathrm{~V}$ & $\mathrm{~N}$ \\
\hline \multicolumn{9}{|l|}{ Plant protection measure } \\
\hline $\begin{array}{l}\text { 1. Identification of insect } \\
\text { pest and disease in host } \\
\text { plants }\end{array}$ & $\begin{array}{c}133 \\
(49.26)\end{array}$ & $\begin{array}{c}136 \\
(50.37)\end{array}$ & $\begin{array}{c}01 \\
(00.37)\end{array}$ & $\begin{array}{c}00 \\
(00.00)\end{array}$ & 672 & 02.49 & I & MN \\
\hline $\begin{array}{l}\text { 2. Insecticide and fungicide } \\
\text { to control insect pest and } \\
\text { diseases }\end{array}$ & $\begin{array}{c}123 \\
(45.56)\end{array}$ & $\begin{array}{c}145 \\
(53.70)\end{array}$ & $\begin{array}{c}02 \\
(00.74)\end{array}$ & $\begin{array}{c}00 \\
(00.00)\end{array}$ & 661 & 02.45 & III & $\mathrm{MN}$ \\
\hline $\begin{array}{l}\text { 3. Use of insecticide and } \\
\text { fungicide }\end{array}$ & $\begin{array}{c}132 \\
(48.89)\end{array}$ & $\begin{array}{c}137 \\
(50.74)\end{array}$ & $\begin{array}{c}01 \\
(00.37)\end{array}$ & $\begin{array}{c}00 \\
(00.00)\end{array}$ & 671 & 02.48 & II & MN \\
\hline $\begin{array}{l}\text { 4. Use of sprayers and } \\
\text { dusters }\end{array}$ & $\begin{array}{c}93 \\
(34.44)\end{array}$ & $\begin{array}{c}153 \\
(56.67)\end{array}$ & $\begin{array}{c}22 \\
(08.15)\end{array}$ & $\begin{array}{c}02 \\
(00.74)\end{array}$ & 607 & 25 & IV & $\mathrm{N}$ \\
\hline
\end{tabular}


Table.4 Training needs in sub-areas of basic practices and handling of eggs and chawki silkworm rearing $(n=270)$

\begin{tabular}{|c|c|c|c|c|c|c|c|c|}
\hline \multirow[t]{2}{*}{ Sub-areas } & \multicolumn{4}{|c|}{ Training need } & \multirow[t]{2}{*}{ TS } & \multirow[t]{2}{*}{ MS } & \multirow[t]{2}{*}{ Rank } & \multirow[t]{2}{*}{$\mathbf{C P}$} \\
\hline & $\begin{array}{c}\mathrm{MN} \\
(\mathrm{F} / \%)\end{array}$ & $\begin{array}{c}\mathrm{N} \\
(\mathrm{F} / \%)\end{array}$ & $\begin{array}{c}\text { SN } \\
(\mathbf{F} / \%)\end{array}$ & $\begin{array}{r}\mathbf{N N} \\
(\mathbf{F} / \%)\end{array}$ & & & & \\
\hline \multicolumn{9}{|c|}{ Basic practices and handling of eggs } \\
\hline $\begin{array}{l}\text { 1. Selection of improved } \\
\text { species of silk worm }\end{array}$ & $\begin{array}{c}164 \\
(60.74)\end{array}$ & $\begin{array}{c}101 \\
(37.41)\end{array}$ & $\begin{array}{c}05 \\
(01.85)\end{array}$ & $\begin{array}{c}00 \\
(00.00)\end{array}$ & 699 & 2.59 & III & MN \\
\hline $\begin{array}{l}\text { 2. Use of equipments and } \\
\text { material required in silk } \\
\text { worm rearing }\end{array}$ & $\begin{array}{c}145 \\
(53.70)\end{array}$ & $\begin{array}{c}114 \\
(42.22)\end{array}$ & $\begin{array}{c}11 \\
(04.08)\end{array}$ & $\begin{array}{c}00 \\
(00.00)\end{array}$ & 674 & 2.49 & IV & MN \\
\hline $\begin{array}{l}\text { 3. Eggs for tasar silk worm } \\
\text { rearing }\end{array}$ & $\begin{array}{c}67 \\
(24.81)\end{array}$ & $\begin{array}{c}157 \\
(58.15)\end{array}$ & $\begin{array}{c}45 \\
(16.67)\end{array}$ & $\begin{array}{c}01 \\
(00.37)\end{array}$ & 560 & 2.07 & V & $\mathrm{N}$ \\
\hline $\begin{array}{l}\text { 4. Ratio of formalin or } \\
\text { H.C.L. solution for } \\
\text { disinfection of tasar } \\
\text { silkworms eggs }\end{array}$ & $\begin{array}{c}198 \\
(73.33)\end{array}$ & $\begin{array}{c}70 \\
(25.93)\end{array}$ & $\begin{array}{c}02 \\
(00.74)\end{array}$ & $\begin{array}{c}00 \\
(00.00)\end{array}$ & 736 & 2.72 & I & MN \\
\hline $\begin{array}{l}\text { 5. Temperature and } \\
\text { humidity for hatching } \\
\text { tasar silkworm eggs }\end{array}$ & $\begin{array}{c}186 \\
(68.89)\end{array}$ & $\begin{array}{c}79 \\
(29.26)\end{array}$ & $\begin{array}{c}05 \\
(01.85)\end{array}$ & $\begin{array}{c}00 \\
(00.00)\end{array}$ & 721 & 2.67 & II & MN \\
\hline \multicolumn{9}{|l|}{ Chawki silkworm rearing } \\
\hline $\begin{array}{l}\text { 1. Brushing of larvae in the } \\
\text { chawki garden }\end{array}$ & $\begin{array}{c}37 \\
(13.70)\end{array}$ & $\begin{array}{c}169 \\
(62.60)\end{array}$ & $\begin{array}{c}59 \\
(21.85)\end{array}$ & $\begin{array}{c}05 \\
(01.85)\end{array}$ & 508 & 01.88 & IV & $\mathrm{N}$ \\
\hline $\begin{array}{l}\text { 2. Brushing of larvae in the } \\
\text { host plants }\end{array}$ & $\begin{array}{c}36 \\
(13.33) \\
\end{array}$ & $\begin{array}{c}151 \\
(55.93)\end{array}$ & $\begin{array}{c}78 \\
(28.89) \\
\end{array}$ & $\begin{array}{c}05 \\
(01.85)\end{array}$ & 488 & 01.81 & VII & $\mathrm{N}$ \\
\hline $\begin{array}{l}\text { 3. Size of nylon net and } \\
\text { methods of covering host } \\
\text { plants in the chawki } \\
\text { garden }\end{array}$ & $\begin{array}{c}103 \\
(38.15)\end{array}$ & $\begin{array}{c}114 \\
(42.22)\end{array}$ & $\begin{array}{c}51 \\
(18.89)\end{array}$ & $\begin{array}{c}02 \\
(00.74)\end{array}$ & 588 & 02.17 & I & $\mathrm{N}$ \\
\hline $\begin{array}{l}\text { 4. Methods of brushing } \\
\text { larvae in chawki garden }\end{array}$ & $\begin{array}{c}56 \\
(20.74) \\
\end{array}$ & $\begin{array}{c}128 \\
(47.41)\end{array}$ & $\begin{array}{c}83 \\
(30.74) \\
\end{array}$ & $\begin{array}{c}03 \\
(01.11)\end{array}$ & 507 & 01.87 & $\mathrm{~V}$ & $\mathrm{~N}$ \\
\hline 5. Size of brushing bag & $\begin{array}{c}63 \\
(23.33)\end{array}$ & $\begin{array}{c}137 \\
(50.74)\end{array}$ & $\begin{array}{c}68 \\
(25.19)\end{array}$ & $\begin{array}{c}02 \\
(00.74)\end{array}$ & 531 & 01.97 & II & $\mathrm{N}$ \\
\hline $\begin{array}{l}\text { 6. Use of brushing bags in } \\
\text { the plants }\end{array}$ & $\begin{array}{c}64 \\
(23.70)\end{array}$ & $\begin{array}{c}126 \\
(46.67)\end{array}$ & $\begin{array}{c}78 \\
(28.89)\end{array}$ & $\begin{array}{c}02 \\
(00.74)\end{array}$ & 522 & 01.93 & III & $\mathrm{N}$ \\
\hline $\begin{array}{l}\text { 7. Stages of silkworms in } \\
\text { chawki garden }\end{array}$ & $\begin{array}{c}54 \\
(20.00)\end{array}$ & $\begin{array}{c}130 \\
(48.15)\end{array}$ & $\begin{array}{c}75 \\
(27.78)\end{array}$ & $\begin{array}{c}11 \\
(04.07)\end{array}$ & 497 & 01.84 & VI & $\mathrm{N}$ \\
\hline
\end{tabular}


Table.5 Training needs in sub-areas of adult stage silkworm rearing and disease and insect control $(n=270)$

\begin{tabular}{|c|c|c|c|c|c|c|c|c|}
\hline \multirow[t]{2}{*}{ Sub-areas } & \multicolumn{4}{|c|}{ Training need } & \multirow[t]{2}{*}{ TS } & \multirow[t]{2}{*}{ MS } & \multirow[t]{2}{*}{ Rank } & \multirow[t]{2}{*}{$\mathbf{C P}$} \\
\hline & $\begin{array}{c}\text { MN } \\
(\mathbf{F} / \%)\end{array}$ & $\begin{array}{c}\mathbf{N} \\
(\mathbf{F} / \%)\end{array}$ & $\begin{array}{r}\text { SN } \\
(\mathbf{F} / \%)\end{array}$ & $\begin{array}{r}\text { NN } \\
(\mathbf{F} / \%)\end{array}$ & & & & \\
\hline \multicolumn{9}{|l|}{ Adult stage silkworm rearing } \\
\hline $\begin{array}{l}\text { 1. Brushing of silkworms in the } \\
\text { host plants }\end{array}$ & $\begin{array}{c}28 \\
(10.37)\end{array}$ & $\begin{array}{c}144 \\
(53.33)\end{array}$ & $\begin{array}{c}85 \\
(31.48)\end{array}$ & $\begin{array}{c}13 \\
(04.82)\end{array}$ & 457 & 01.69 & IV & $\mathrm{N}$ \\
\hline $\begin{array}{l}\text { 2. Transfer of silkworms in } \\
\text { economic plants from chawki } \\
\text { garden }\end{array}$ & $\begin{array}{c}35 \\
(12.96)\end{array}$ & $\begin{array}{c}140 \\
(51.85)\end{array}$ & $\begin{array}{c}79 \\
(29.26)\end{array}$ & $\begin{array}{c}16 \\
(05.93)\end{array}$ & 464 & 01.72 & III & $\mathrm{N}$ \\
\hline $\begin{array}{l}\text { 3. Foliar spray of urea solution } \\
\text { before transfer of silk worms } \\
\text { on the host plants }\end{array}$ & $\begin{array}{c}77 \\
(28.52)\end{array}$ & $\begin{array}{c}150 \\
(55.56)\end{array}$ & $\begin{array}{c}42 \\
(15.55)\end{array}$ & $\begin{array}{c}01 \\
(00.37)\end{array}$ & 573 & 02.12 & II & $\mathrm{N}$ \\
\hline $\begin{array}{l}\text { 4. Disinfections of field by } \\
\text { spreading of lime rand } \\
\text { bleaching powder before } \\
\text { transfer of silkworms. }\end{array}$ & $\begin{array}{c}148 \\
(54.81)\end{array}$ & $\begin{array}{c}114 \\
(42.22)\end{array}$ & $\begin{array}{c}07 \\
(02.60)\end{array}$ & $\begin{array}{c}01 \\
(00.37)\end{array}$ & 679 & 02.51 & I & $\mathrm{MN}$ \\
\hline \multicolumn{9}{|l|}{ Disease and insect control } \\
\hline $\begin{array}{l}\text { 1. About attack of parasite and } \\
\text { predators at larval and adult } \\
\text { stage of silkworm rearing }\end{array}$ & $\begin{array}{c}164 \\
(60.74)\end{array}$ & $\begin{array}{c}77 \\
(28.52)\end{array}$ & $\begin{array}{c}06 \\
(02.22)\end{array}$ & $\begin{array}{c}23 \\
(08.52)\end{array}$ & 652 & 02.41 & VI & $\mathrm{MN}$ \\
\hline $\begin{array}{l}\text { 2. About attack of insect pest and } \\
\text { diseases at larval and adult } \\
\text { stage of silkworm rearing }\end{array}$ & $\begin{array}{c}188 \\
(69.63)\end{array}$ & $\begin{array}{c}80 \\
(29.63)\end{array}$ & $\begin{array}{c}01 \\
(00.37)\end{array}$ & $\begin{array}{c}01 \\
(00.37)\end{array}$ & 725 & 02.68 & IV & $\mathrm{MN}$ \\
\hline $\begin{array}{l}\text { 3. Ways to check pests (e.i. ants, } \\
\text { lezzard, rates, etc.) to attack in } \\
\text { the host plants }\end{array}$ & $\begin{array}{c}174 \\
(64.45)\end{array}$ & $\begin{array}{c}83 \\
(30.74)\end{array}$ & $\begin{array}{c}12 \\
(04.44)\end{array}$ & $\begin{array}{c}01 \\
(00.37)\end{array}$ & 700 & 02.59 & V & MN \\
\hline $\begin{array}{l}\text { 4. Name, quantity and method of } \\
\text { using insecticide and fungicide }\end{array}$ & $\begin{array}{c}203 \\
(75.19)\end{array}$ & $\begin{array}{c}67 \\
(24.81)\end{array}$ & $\begin{array}{c}00 \\
(00.00)\end{array}$ & $\begin{array}{c}00 \\
(00.00)\end{array}$ & 743 & 02.75 & II & $\mathrm{MN}$ \\
\hline $\begin{array}{l}\text { 5. Time of using insecticide and } \\
\text { fungicide }\end{array}$ & $\begin{array}{c}199 \\
(73.70)\end{array}$ & $\begin{array}{c}71 \\
(26.30)\end{array}$ & $\begin{array}{c}00 \\
(00.00)\end{array}$ & $\begin{array}{c}00 \\
(00.00)\end{array}$ & 739 & 02.73 & III & $\mathrm{MN}$ \\
\hline $\begin{array}{l}\text { 6. Use of bio-pesticide and } \\
\text { insecticide }\end{array}$ & $\begin{array}{c}221 \\
(81.85)\end{array}$ & $\begin{array}{c}49 \\
(18.15)\end{array}$ & $\begin{array}{c}00 \\
(00.00)\end{array}$ & $\begin{array}{c}00 \\
(00.00)\end{array}$ & 761 & 02.81 & I & $\mathrm{MN}$ \\
\hline 7. Use of sprayers and dusters & $\begin{array}{c}76 \\
(28.15)\end{array}$ & $\begin{array}{c}170 \\
(62.96)\end{array}$ & $\begin{array}{c}22 \\
08.15\end{array}$ & $\begin{array}{c}02 \\
(00.74)\end{array}$ & 590 & 02.18 & VIII & $\mathrm{N}$ \\
\hline $\begin{array}{l}\text { 8. Control attack of insects in the } \\
\text { storage house }\end{array}$ & $\begin{array}{c}87 \\
(32.22)\end{array}$ & $\begin{array}{c}156 \\
(57.78)\end{array}$ & $\begin{array}{c}23 \\
(08.52)\end{array}$ & $\begin{array}{c}04 \\
(01.48)\end{array}$ & 596 & 02.21 & VII & $\mathrm{N}$ \\
\hline
\end{tabular}


Table.6 Training needs in sub-areas of harvesting and storage, processing of cocoons $(n=270)$

\begin{tabular}{|c|c|c|c|c|c|c|c|c|}
\hline \multirow[t]{2}{*}{ Sub-areas } & \multicolumn{4}{|c|}{ Training need } & \multirow[t]{2}{*}{ TS } & \multirow[t]{2}{*}{ MS } & \multirow[t]{2}{*}{ Rank } & \multirow[t]{2}{*}{$\mathbf{C P}$} \\
\hline & $\begin{array}{c}\text { MN } \\
(\mathbf{F} / \%)\end{array}$ & $\begin{array}{c}\mathbf{N} \\
(\mathbf{F} / \%)\end{array}$ & $\begin{array}{c}\text { SN } \\
(\mathbf{F} / \%)\end{array}$ & $\begin{array}{c}\mathbf{N N} \\
(\mathbf{F} / \%)\end{array}$ & & & & \\
\hline \multicolumn{9}{|c|}{ Harvesting and storage of cocoons } \\
\hline $\begin{array}{l}\text { 1. Best time for harvesting } \\
\text { of cocoon }\end{array}$ & $\begin{array}{c}17 \\
(06.30)\end{array}$ & $\begin{array}{c}22 \\
(08.15)\end{array}$ & $\begin{array}{c}36 \\
(13.33)\end{array}$ & $\begin{array}{c}195 \\
(72.22)\end{array}$ & 131 & 00.48 & VI & NN \\
\hline $\begin{array}{l}\text { 2. Methods of cutting } \\
\text { cocoons }\end{array}$ & $\begin{array}{c}16 \\
(05.93)\end{array}$ & $\begin{array}{c}22 \\
(08.15)\end{array}$ & $\begin{array}{c}48 \\
(17.77)\end{array}$ & $\begin{array}{c}184 \\
(68.15)\end{array}$ & 140 & 00.51 & V & $\mathrm{NN}$ \\
\hline $\begin{array}{l}\text { 3. Selection of healthy } \\
\text { cocoons }\end{array}$ & $\begin{array}{c}30 \\
(11.11)\end{array}$ & $\begin{array}{c}81 \\
(30.00)\end{array}$ & $\begin{array}{c}91 \\
(33.70)\end{array}$ & $\begin{array}{c}68 \\
(25.19)\end{array}$ & 343 & 01.27 & IV & $\mathrm{SN}$ \\
\hline $\begin{array}{l}\text { 4. Preparation of chain of } \\
\text { cocoons }\end{array}$ & $\begin{array}{c}31 \\
(11.48)\end{array}$ & $\begin{array}{c}104 \\
(38.52)\end{array}$ & $\begin{array}{c}96 \\
(35.56)\end{array}$ & $\begin{array}{c}39 \\
(14.44)\end{array}$ & 397 & 01.47 & II & $\mathrm{SN}$ \\
\hline $\begin{array}{l}\text { 5. Methods of storage of } \\
\text { cocoons after harvesting }\end{array}$ & $\begin{array}{c}32 \\
(11.85)\end{array}$ & $\begin{array}{c}80 \\
(29.63)\end{array}$ & $\begin{array}{c}121 \\
(44.82)\end{array}$ & $\begin{array}{c}37 \\
(13.70)\end{array}$ & 377 & 01.39 & III & $\mathrm{SN}$ \\
\hline $\begin{array}{l}\text { 6. Commercial storage of } \\
\text { cocoons }\end{array}$ & $\begin{array}{c}39 \\
(14.44)\end{array}$ & $\begin{array}{c}100 \\
(37.04)\end{array}$ & $\begin{array}{c}94 \\
(34.82)\end{array}$ & $\begin{array}{c}37 \\
(13.70)\end{array}$ & 411 & 01.52 & $\mathrm{I}$ & $\mathrm{N}$ \\
\hline \multicolumn{9}{|l|}{ Processing of cocoons } \\
\hline 1. Grading of cocoons & $\begin{array}{c}176 \\
(65.19)\end{array}$ & $\begin{array}{c}89 \\
(32.96)\end{array}$ & $\begin{array}{c}04 \\
(01.48)\end{array}$ & $\begin{array}{c}01 \\
(00.37)\end{array}$ & 710 & 02.63 & III & $\mathrm{MN}$ \\
\hline 2. Boiling of cocoons & $\begin{array}{c}129 \\
(47.78)\end{array}$ & $\begin{array}{c}80 \\
(29.63)\end{array}$ & $\begin{array}{c}53 \\
(19.63)\end{array}$ & $\begin{array}{c}08 \\
(2.96)\end{array}$ & 600 & 02.22 & IV & $\mathrm{N}$ \\
\hline 3. Reeling of cocoons & $\begin{array}{c}202 \\
(74.82)\end{array}$ & $\begin{array}{c}63 \\
(23.33)\end{array}$ & $\begin{array}{c}00 \\
(00.00)\end{array}$ & $\begin{array}{c}05 \\
(01.85)\end{array}$ & 732 & 02.71 & II & $\mathrm{MN}$ \\
\hline 4. Methods of reeling & $\begin{array}{c}202 \\
(74.82)\end{array}$ & $\begin{array}{c}64 \\
(23.70)\end{array}$ & $\begin{array}{c}01 \\
(00.37)\end{array}$ & $\begin{array}{c}03 \\
(01.11)\end{array}$ & 735 & 02.72 & I & MN \\
\hline 5. Preparation of products & $\begin{array}{c}201 \\
(74.45)\end{array}$ & $\begin{array}{c}63 \\
(23.33)\end{array}$ & $\begin{array}{c}03 \\
(01.11)\end{array}$ & $\begin{array}{c}03 \\
(01.11)\end{array}$ & 732 & 02.71 & II & $\mathrm{MN}$ \\
\hline
\end{tabular}

Table.7 Training needs of sericulturist in the major areas of tasar silk cultivation $(n=270)$

\begin{tabular}{|c|c|c|c|c|c|c|c|c|}
\hline \multirow[t]{2}{*}{ Area of training } & \multicolumn{4}{|c|}{ Training need } & \multirow[t]{2}{*}{ TS } & \multirow[t]{2}{*}{ MS } & \multirow[t]{2}{*}{ Rank } & \multirow[t]{2}{*}{$\mathbf{C P}$} \\
\hline & $\begin{array}{c}\text { MN } \\
(\mathbf{F} / \%)\end{array}$ & $\begin{array}{c}\mathbf{N} \\
(\mathbf{F} / \%)\end{array}$ & $\begin{array}{c}\mathrm{SN} \\
(\mathrm{F} / \%)\end{array}$ & $\begin{array}{c}\mathrm{NN} \\
(\mathrm{F} / \%)\end{array}$ & & & & \\
\hline \multicolumn{9}{|l|}{ Host plant cultivation } \\
\hline$>$ Land preparation & $\frac{13}{(04.81)}$ & $\begin{array}{c}50 \\
(18.52)\end{array}$ & $\begin{array}{c}86 \\
(31.85)\end{array}$ & $\frac{121}{(44.82)}$ & 224 & 00.83 & V & SN \\
\hline$>$ Plant propagation & $\begin{array}{c}22 \\
(08.15)\end{array}$ & $\begin{array}{c}79 \\
(29.26)\end{array}$ & $\begin{array}{c}70 \\
(25.92)\end{array}$ & $\begin{array}{c}99 \\
(36.67)\end{array}$ & 294 & 01.09 & IV & SN \\
\hline$>$ Handling of plants & $\begin{array}{c}17 \\
(06.30)\end{array}$ & $\begin{array}{c}111 \\
(41.11)\end{array}$ & $\begin{array}{c}54 \\
(20.00)\end{array}$ & $\begin{array}{c}88 \\
(32.59)\end{array}$ & 327 & 1.21 & III & $\mathrm{SN}$ \\
\hline $\begin{array}{l}\text { Application FYM and } \\
\text { fertilizers }\end{array}$ & $\begin{array}{c}85 \\
(31.48)\end{array}$ & $\begin{array}{c}163 \\
(60.37)\end{array}$ & $\begin{array}{c}20 \\
(07.41)\end{array}$ & $\begin{array}{c}02 \\
(00.74)\end{array}$ & 601 & 02.22 & II & $\mathrm{N}$ \\
\hline$>$ Plant protection & $\begin{array}{c}120 \\
(44.45)\end{array}$ & $\begin{array}{c}143 \\
(52.96)\end{array}$ & $\begin{array}{c}06 \\
(02.22)\end{array}$ & $\begin{array}{c}01 \\
(00.37)\end{array}$ & 652 & 02.41 & I & $\mathrm{MN}$ \\
\hline
\end{tabular}




\begin{tabular}{|c|c|c|c|c|c|c|c|c|}
\hline \multicolumn{9}{|l|}{ Silk worm rearing } \\
\hline $\begin{array}{l}\text { Basic practices and } \\
\text { handling of eggs }\end{array}$ & $\begin{array}{c}152 \\
(56.30)\end{array}$ & $\begin{array}{c}104 \\
(38.52)\end{array}$ & $\begin{array}{c}13 \\
(04.81)\end{array}$ & $\begin{array}{c}01 \\
(00.37)\end{array}$ & 677 & 02.50 & III & $\mathrm{MN}$ \\
\hline $\begin{array}{l}\text { Chawki silkworm } \\
\text { rearing }\end{array}$ & $\begin{array}{c}59 \\
(21.85)\end{array}$ & $\begin{array}{c}137 \\
(50.74)\end{array}$ & $\begin{array}{c}70 \\
(25.93)\end{array}$ & $\begin{array}{c}04 \\
(01.48)\end{array}$ & 521 & 01.93 & $\mathrm{~V}$ & $\mathrm{~N}$ \\
\hline $\begin{array}{l}\text { Adult stage silkworm } \\
\text { rearing }\end{array}$ & $\begin{array}{c}72 \\
(26.67)\end{array}$ & $\begin{array}{c}137 \\
(50.74)\end{array}$ & $\begin{array}{c}53 \\
(19.63)\end{array}$ & $\begin{array}{c}08 \\
(02.96)\end{array}$ & 583 & 02.01 & IV & $\mathrm{N}$ \\
\hline $\begin{array}{l}>\text { Disease and Insect } \\
\text { Control }\end{array}$ & $\begin{array}{c}164 \\
(60.74)\end{array}$ & $\begin{array}{c}94 \\
(34.82)\end{array}$ & $\begin{array}{c}08 \\
(02.96)\end{array}$ & $\begin{array}{c}04 \\
(01.48)\end{array}$ & 688 & 02.55 & II & $\mathrm{MN}$ \\
\hline $\begin{array}{l}\text { Harvesting and storage } \\
\text { of cocoons }\end{array}$ & $\begin{array}{c}28 \\
(10.37)\end{array}$ & $\begin{array}{c}68 \\
(25.19)\end{array}$ & $\begin{array}{c}81 \\
(30.00)\end{array}$ & $\begin{array}{c}93 \\
(34.44)\end{array}$ & 301 & 01.11 & VI & SN \\
\hline$>$ Processing of cocoons & $\begin{array}{c}182 \\
(67.41)\end{array}$ & $\begin{array}{c}72 \\
(26.67)\end{array}$ & $\begin{array}{c}12 \\
(04.44)\end{array}$ & $\begin{array}{c}04 \\
(01.48)\end{array}$ & 702 & 02.60 & I & $\mathrm{MN}$ \\
\hline
\end{tabular}

Note: $\mathrm{MN}=$ Most Needed, $\mathrm{N}=$ Needed, $\mathrm{SN}=$ Somewhat Needed, NN $=$ Not Needed, TS $=$ Total Score, MS $=$ Mean Score, $\mathrm{CP}=$ Category of Preferences

Table.8 Distribution of respondents according to their overall training need about tasar silk cultivation $(\mathrm{n}=270)$

\begin{tabular}{|l|c|c|}
\hline \multicolumn{1}{|c|}{ Particulars } & Frequency & Percentage \\
\hline Not needed (No score) & 39 & 14.45 \\
\hline Somewhat needed (1 to 65 scores) & 43 & 15.92 \\
\hline Needed (66 to 130 scores) & 105 & 38.89 \\
\hline Most needed (Above 130 scores) & 83 & 30.74 \\
\hline \multicolumn{1}{|c|}{ Total } & $\mathbf{2 7 0}$ & $\mathbf{1 0 0 . 0 0}$ \\
\hline
\end{tabular}

Table.9 Perceived preferences of farmers regarding organization of training programme $(\mathrm{n}=270)$

\begin{tabular}{|l|c|c|}
\hline \multicolumn{1}{|c|}{ Particulars } & Frequency & Percentage \\
\hline Place of training & & \\
\hline 1. At the village level & 157 & 58.15 \\
\hline 2. Exhibition centre & 71 & 26.30 \\
\hline $\begin{array}{l}\text { 3. At the nearest research extension centre/ departmental } \\
\text { farm }\end{array}$ & 19 & 07.04 \\
\hline 4. At the research station & 23 & 08.51 \\
\hline $\begin{array}{l}\text { Duration of training } \\
\text { 1. One week }\end{array}$ & 176 & \\
\hline 2. Two weeks & 76 & 65.18 \\
\hline 3. One month & 18 & 28.15 \\
\hline Season of training & & 06.67 \\
\hline 1. Pre crop season & 265 & \\
\hline 2. Crop season & 05 & 98.15 \\
\hline 3. Post crop season & 00 & 01.85 \\
\hline Nature of training & & \\
\hline
\end{tabular}




\begin{tabular}{|l|c|c|}
\hline 1. Basic training & 22 & 08.15 \\
\hline 2. Practical utility training & 248 & 91.85 \\
\hline Facilities of training* & & \\
\hline 1. Free boarding & 107 & 39.63 \\
\hline 2. Provide stipend & 218 & 80.74 \\
\hline 3. Provide transportation & 110 & 40.74 \\
\hline 4. Exposure visit & 35 & 12.96 \\
\hline 5. Teaching through audio visual aids & 84 & 31.11 \\
\hline Trainers language & & \\
\hline 1. Local language & 206 & 76.30 \\
\hline 2. Hindi & 64 & 23.70 \\
\hline 3. English & 00 & 00.00 \\
\hline Methods of training & & \\
\hline 1. Demonstration & 92 & 34.07 \\
\hline 2. Lectures & 69 & 25.55 \\
\hline 3. Group discussion/ conference & 44 & 16.30 \\
\hline 4. Audio visual aids/ Kisan mela/ Exhibitions/ Debates etc. & 65 & 24.08 \\
\hline Number of trainees & & \\
\hline 1. Up to 20 trainees & 97 & 35.93 \\
\hline 2. 21 to 30 trainees & 105 & 38.89 \\
\hline 3. 31 to 40 trainees & 51 & 18.89 \\
\hline 4. 40 to 50 trainees & 14 & 05.18 \\
\hline 5. Above 50 trainees & 03 & 01.11 \\
\hline Number of training in a year & & \\
\hline 1. Once in a year & 48 & 17.78 \\
\hline 2. Twice in a year & 156 & 57.78 \\
\hline 3. Thrice in a year & 64 & 23.70 \\
\hline 4. More than thrice in a year & 02 & 00.74 \\
\hline
\end{tabular}

As regards to the duration of training, 65.18 per cent of the respondents suggested for arranging one weeks training programme against two weeks training period suggested by 28.15 per cent of the respondents. On the other hand, very few $(8.51 \%)$ respondents suggested for one month training period. Mech et al., (2013) had doing almost similar study on training nee of rural women in Eri culture in Assam Province. Reddy (2006), Geetha and Devi (2008), Gurav et al., (2010), Nain and Chandel (2010) and Paswan et al., (2013) gives almost similar findings in their studies.
Season is one of the important aspects of training especially for all kind of sericulturists. Tasar silkworm rearing is being used as main occupation and doing mostly during leisure period of time of the sericulturist. The data regarding preference of season of training, majority of the respondents (98.15\%) expressed the pre-crop season as ideal for training. Crop season was chosen by only 1.85 per cent of the respondents. None of the respondent preferred post crop season training. Mech et al., (2013) had reported almost similar findings on training need of rural women towards Eri culture in Assam Province. 
Data in respect of nature of training indicated that the majority of the respondents (91.85\%) expressed the need of practical training instead of basic training $(8.15 \%)$. There is no doubt that practical utility training would definitely add utmost to their knowledge and skills that can be useful in the field efficiency. Rahmathulla et al., (2006), Nain and Chandel (2010), Vindhya et al., (2012) and Mech et al., (2013) had given almost similar findings their studies.

An effective training programme is largely depend on facilities of training provides to the trainees. The present study shown that, majority $(80.74 \%)$ of the respondents stated that for the stipend during training should be provided, followed by 40.74 per cent respondents required transport facilities. About us 39.63 per cent of the respondents required free boarding facilities, 31.11 per cent of the respondents required audio-visual teaching facilities, only 12.96 per cent of the respondents stated notably for the facilities of exposure visit. The above facilities make trainings more comfortable and effective. Mech et al., (2013) had given almost similar findings their study.

Language is considered as a key aspect of the farmers training programme because educational status of the farmers is generally low and sometime frequency of illiterate farmers is high. About 76 per cent of the respondents suggested local language followed by 23.70 per cent of the respondents suggested hindi language as the communicating medium between trainers and trainees. None of the sericulturist preferred english as teaching medium during training. Mech et al., (2013) had given almost similar findings their study.

As regards to method of training, 34.07 per cent of the respondents suggested demonstration, followed by lectures
(22.55\%), audio-visual aids/ kisan mela/ exhibition/ debates etc. (24.08\%) and only 16.30 per cent of the respondents suggested group discussion/conference for training. Paswan et al., (2013) gives almost similar findings in their study.

Data regarding number of training, maximum number of respondents $(38.89 \%)$ said 21 to 30 trainees is suitable for best training followed by 35.93 per cent of the respondents said up to 20 trainees, 18.89 per cent of the respondents said 31 to 40 trainees. On the other hand 5.18 per cent and 1.11 per cent of the respondents said 40 to 50 trainees and above 50 trainees were suitable for best training programme. Nain and Chandel (2010) and Paswan et al., (2013) gives almost similar findings in their study.

Data in respect of number of training in a year, majority $(57.78 \%)$ of the respondents suggested two training are suitable in a year, followed by 23.70 per cent of the respondents said three training in a year is suitable, 17.78 per cent of the respondents said only one training in a year is suitable while only 00.74 per cent of the respondents said more than three training in a year is suitable.

In conclusion it is evident from the findings that a sizeable chunk of silkworm rearing farmers are already attended training programmes. The training need of the sericulturists on plant protection measure, processing of cocoons and disease and insect pest control in silkworm are more prominent. The extent of training need indicated that most of the sericulturists extremely needed the training programme of improved tasar silk cultivation practices. The study also inferred that sericulturists mostly desired training at village level for a period of one week at precrop season and also practical utility training with facilities like stipend, transportation, free boarding and teaching through demonstration 
and lecture method using local language. Proper implication of all suggested area is an urgent need for tasar silkworm rearing farmers for increasing its productivity and profitability.

\section{References}

Chawang, J.K. and Jha, K.K. 2010. Training Needs of Paddy Cultivators in Nagaland. Indian Res. J. Ext. Edu. 10 (1): 74-77.

Dewangan, S.K. 2010. Sericulture - A Tool of Eco-System Checking Through Tribal. Journal of Environmental Research and Development, 6(1).

Geetha, G.S. and Devi, G.R.G. 2008. Technology adoption and training needs of sericulture farmers-a case study in NGO. Indian Journal of Agricultural Research, 42(3): 157 -163.

Gurav, K.V., Khalache, P.G. and Thorat, K.S. 2010. Training Needs of Agriculture Assistants from Single Window System in Maharashtra. Asian Journal of Extension Education, 28 (1\&2): 119122.

Hanumappa, H.G. and Erappa, S. 1985. Economic issues in sericulture: study of Karnataka. Econ. Political weekly, 20(31): 3122-3224.

Kushwaha, R.V. and Singhavi, N.R. 2013. Extent of adoption of improved sericultural practices by the sericulturists of Buldhana district of Maharashtra. Agriculture Update, 8(3): 469-471.

Lakshmanan, S., Mallikarjuna, B., Rao, R.G., Jayaram, H. and Geetadavi, R.G. 1998. Studies on adoption of sericultural innovation at farmers level in Tamil Nadu: An empirical analysis. Indian J. Seric. 37(1): 44-47.

Manju, S. 1997. A study on sericultural practices and marketing problems faced by the sericulturists of Belgaum
District. M. Sc. (Ag.) Thesis, University of Agricultural Sciences, Dharwad (Karnataka).

Mech, D., Handique, P.K. and Barman, H. 2010. Training need of rural women in Eri culture in Assam province. Global Journal of Human Social Science, 10(7): 5-7.

Nain, M.S. and Chandel, S.S. 2010. Determinants of Farmers' Training Needs in Agri-horti Farming System: A Study of J\&K State. Journal of Community Mobilization and Sustainable Development, 5(2): 023027.

Paswan, A., Sinha, K.K., Sriwastava, J.N. and Paswan, A.K. 2013. Need based trainings and constraints of banana growers in Bhagalpur, Bihar. Agriculture Update, 8(3): 509-513.

Patel, G.P.D. and Veerbhadraiah, V. 1987. Mysore J. Agric. Sci. 21: 224.

Prasad, V.L.M., Naika, R., Usha, R. and Raj, M.P.G. 2010. Training needs and source consultancy pattern of commercial chawki rearing centre owners in Kolar district. Agriculture Update, 5(3/4): 325-327.

Rahmathulla, V.K., Srinivasa, G., Vindhya, G.S. and Rajan, R.K. 2006. Assessment of sericultural technology training programme - an analysis of sericulture trainees. Indian Journal of Sericulture, 45(1): 11-14.

Ranganatha, A.D. et al., 2003. In: Abstracts of National Conference Tropical Sericulture for Global Competitiveness, 5-7 Nov., 2003, C.S.R. and T.I., Mysore, pp. 153-154.

Reddy, V.S. 2006. Knowledge and adoption of integrated pest management practices among vegetable growers of Gadag district in North-Karnataka. M.Sc. (Ag.) Thesis, University of Agricultural Sciences, Dharwad (Karnataka). pp. 4647. 
Shivamurthy, M. et al., 2003. In: Abstracts of National Conference Tropical Sericulture for Global Competitiveness, 5-7 Nov., 2003, C.S.R. and T.I., Mysore, pp. 140.

Verma, H.K., Patel, B.M., Patel, J.K. and Gulkari, K.D. 2013. Training needs assessment of marigold growers. Agriculture Update, 8(1): 257-259.
Vindhya, G.S., Devi, G.R.G., Rao, D.M. and Ram. 2012. Identification of training needs for farmers for adoption of sericulture technologies: a case study in Mysore District. Indian Journal of Training and Development, 42(1): 4657.

\section{How to cite this article:}

Kedar Nath Yadaw and Sharma, M. L. 2021. Training Need Assessment of Tasar Silkworm Rearing Farmers in Chhattisgarh. Int.J.Curr.Microbiol.App.Sci. 10(01): 3067-3082. doi: https://doi.org/10.20546/ijcmas.2021.1001.357 